\title{
Facile synthesis of 4,4'-bis-sydnones
}

\author{
Yiwen Yang,* Chunxin Lu, and Wei Zhong \\ College of Biological, Chemical Sciences, and Engineering, Jiaxing University, \\ Jiahang Road 118, Jiaxing 314001, China \\ E-mail:yangyiwen@mail.zjxu.edu.cn
}

DOI: http://dx.doi.org/10.3998/ark.5550190.p009.180

\begin{abstract}
An efficient method is developed for direct dehydrogenative $\mathrm{C}-\mathrm{C}$ coupling of 3-arylsydnones in the presence of palladium(II) acetate catalyst under oxygen. The reaction proceeds efficiently and leads to the formation of bis-sydnones in high yields
\end{abstract}

Keywords: 3-Arylsydnones, palladium(II) acetate, $\mathrm{C}-\mathrm{H}$ bond activation, 4,4'-bis-sydnones

\section{Introduction}

Sydnones, unique, dipolar, and five-membered heterocycles, ${ }^{1}$ are of interest because of their diverse biological activities, such as antimicrobial, ${ }^{2}$ anti-inflammatory, ${ }^{3}$ antitumor, ${ }^{4}$ antimalarial, ${ }^{5}$ analgesic, and antipyretic. ${ }^{6}$ These compounds are also known for their potential applications in therapeutic agents, ${ }^{7-8}$ liquid crystals, ${ }^{9-10}$ and electrolytic solvents. ${ }^{11}$ Previous research on the synthesis of bis-sydnone focused on the use of 4-halosydnone or mercuric chloride derivatives of sydnone as starting materials, ${ }^{12-13}$ which often need to be synthesized. These methods leads generally to low yields. Specifically, the multi-step synthesis of bis-(3phenylsydnone) which involved the use of butyllithium to form unstable lithiosydnone species and the addition of copper (I) bromide or copper (II) chloride under harsh condition $\left(-78{ }^{\circ} \mathrm{C}\right.$ ) resulted in low yield (25\%) products. ${ }^{14}$ Recently, a series of new synthetic methods for the direct functionalization of 3-arylsydnones have been developed. ${ }^{15-17}$ However, there is still no literature reporting the preparation of bis-sydnones. Therefore, it is desirable to develop a convenient and practical synthetic route for the synthesis of them.

$\mathrm{C}-\mathrm{H}$ bond activation catalyzed by transition metals and $\mathrm{C}-\mathrm{C}$ bond formation, which features high atom economy and bond formation efficiency, have attracted much attention in recent years. ${ }^{18-21}$ Direct cross-dehydrogenative coupling of $\mathrm{C}-\mathrm{H}$ bonds, which avoids prefunctionalization of the substrates has been successfully used to construct organic molecules. ${ }^{22-23}$ 
In this paper, we report a novel method for the synthesis of bis-sydnones (2) via direct crossdehydrogenative coupling of 3-arylsydnones (1) in the presence of Pd (II) catalyst under oxygen (Scheme 1).

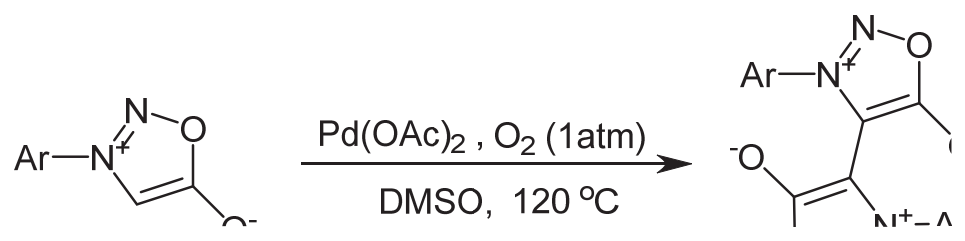

Scheme 1. Synthesis of bis-sydnones (2).

\section{Results and Discussion}

To establish the optimal reaction conditions, we selected 3-(4-methoxyphenyl)sydnone (1a) as the model substrate. A mixture of $1 \mathbf{a}(0.3 \mathrm{mmol}), \mathrm{Pd}(\mathrm{OAc})_{2}(0.03 \mathrm{mmol})$, and $\mathrm{Cu}(\mathrm{OAc})_{2}(0.6$ mmol) was mixed with $2 \mathrm{~mL}$ of DMSO in a sealed tube and stirred in an oil bath at $120{ }^{\circ} \mathrm{C}$. After $16 \mathrm{~h}$, product $\mathbf{2 a}$ was obtained in $93 \%$ yield (Table 1, entry 1 ). The effects of different catalysts and oxidants in different solvents at various reaction temperatures on the formation of $\mathbf{2 a}$ were investigated. Table 1 shows that using toluene, 1,2-dichloroethane (DCE), 1,4-dioxane, $N, N$ dimethylformamide (DMF), and acetonitrile as solvents, instead of DMSO, the yield of 2a (Table 1, entries 2-6) was decreased. Thus, DMSO was determined to be the best solvent. Oxidant screening showed that an improved chemical yield could be obtained by using $\mathrm{O}_{2}$ ( $1 \mathrm{~atm}$ ) as the oxidant, whereas other oxidants, such as $\mathrm{K}_{2} \mathrm{~S}_{2} \mathrm{O}_{8}, \mathrm{Ag}_{2} \mathrm{CO}_{3}, \mathrm{AgOAc}$, and benzoquinone were less effective (Table 1, entries 8-12). When no oxidant was used in the reaction, the yield decreased to $63 \%$ (Table 1, entry 7 ). Therefore, $\mathrm{O}_{2}(1 \mathrm{~atm})$ was chosen as the ideal oxidant. The effect of various dosage of $\mathrm{Pd}(\mathrm{OAc})_{2}$ was further investigated. When the dosage of $\mathrm{Pd}(\mathrm{OAc})_{2}$ was reduced to 0.05 and 0.02 eq., the yield was decreased (Table 1, entries 13-14). Different reaction temperatures were also examined. When the reaction temperature was decreased, the yield dropped dramatically (Table 1, entries 15-17). The effects of different catalysts on 2a formation were also evaluated. 2a was not obtained when no catalyst was used in the reaction or when the catalyst $\mathrm{Pd}(\mathrm{OAc})_{2}$ was changed to $\mathrm{CuI}$ and $\mathrm{FeCl}_{3}$ (Table 1, entries 18-21). Considering these findings, we established the optimal reaction conditions as follows: 0.1 eq. $\mathrm{Pd}(\mathrm{OAc})_{2}$ catalyst, DMSO solvent, $120{ }^{\circ} \mathrm{C}$ reaction temperature, and $16 \mathrm{~h}$ reaction time under $\mathrm{O}_{2}(1 \mathrm{~atm})$ atmosphere (Table 1, entry 12). 
Table 1. Screening for optimal reaction conditions ${ }^{\mathrm{a}}$



\begin{tabular}{|c|c|c|c|c|c|}
\hline \multirow[b]{2}{*}{ Entry } & \multicolumn{2}{|l|}{$1 \mathrm{a}$} & \multicolumn{2}{|c|}{$2 a$} & \multirow[b]{2}{*}{$\begin{array}{c}\text { Yield of } 2 \mathrm{a} \\
(\%)^{\mathrm{b}}\end{array}$} \\
\hline & Catalyst( 0.1 eq.) & Oxidant(2 eq.) & Solvent & $\mathrm{T}\left({ }^{\mathrm{o}} \mathrm{C}\right)$ & \\
\hline 1 & $\mathrm{Pd}(\mathrm{OAc})_{2}$ & $\mathrm{Cu}(\mathrm{OAc})_{2}$ & DMSO & 120 & 93 \\
\hline 2 & $\mathrm{Pd}(\mathrm{OAc})_{2}$ & $\mathrm{Cu}(\mathrm{OAc})_{2}$ & toluene & 120 & 33 \\
\hline 3 & $\mathrm{Pd}(\mathrm{OAc})_{2}$ & $\mathrm{Cu}(\mathrm{OAc})_{2}$ & DCE & 120 & 26 \\
\hline 4 & $\mathrm{Pd}(\mathrm{OAc})_{2}$ & $\mathrm{Cu}(\mathrm{OAc})_{2}$ & 1,4-dioxane & 120 & 55 \\
\hline 5 & $\mathrm{Pd}(\mathrm{OAc})_{2}$ & $\mathrm{Cu}(\mathrm{OAc})_{2}$ & DMF & 120 & 12 \\
\hline 6 & $\mathrm{Pd}(\mathrm{OAc})_{2}$ & $\mathrm{Cu}(\mathrm{OAc})_{2}$ & acetonitrile & 120 & 37 \\
\hline 7 & $\mathrm{Pd}(\mathrm{OAc})_{2}$ & none & DMSO & 120 & 63 \\
\hline 8 & $\mathrm{Pd}(\mathrm{OAc})_{2}$ & $\mathrm{~K}_{2} \mathrm{~S}_{2} \mathrm{O}_{8}$ & DMSO & 120 & 74 \\
\hline 9 & $\mathrm{Pd}(\mathrm{OAc})_{2}$ & $\mathrm{Ag}_{2} \mathrm{CO}_{3}$ & DMSO & 120 & 71 \\
\hline 10 & $\mathrm{Pd}(\mathrm{OAc})_{2}$ & $\mathrm{AgOAc}$ & DMSO & 120 & 68 \\
\hline 11 & $\mathrm{Pd}(\mathrm{OAc})_{2}$ & Benzoquinone & DMSO & 120 & 26 \\
\hline 12 & $\operatorname{Pd}(\mathrm{OAc})_{2}$ & $\mathrm{O}_{2}(1 \mathrm{~atm})$ & DMSO & 120 & 95 \\
\hline 13 & $\mathrm{Pd}(\mathrm{OAc})_{2}(0.05)$ & $\mathrm{O}_{2}(1 \mathrm{~atm})$ & DMSO & 120 & 52 \\
\hline 14 & $\mathrm{Pd}(\mathrm{OAc})_{2}(0.02)$ & $\mathrm{O}_{2}(1 \mathrm{~atm})$ & DMSO & 120 & 40 \\
\hline 15 & $\mathrm{Pd}(\mathrm{OAc})_{2}$ & $\mathrm{O}_{2}(1 \mathrm{~atm})$ & DMSO & 90 & 80 \\
\hline 16 & $\mathrm{Pd}(\mathrm{OAc})_{2}$ & $\mathrm{O}_{2}(1 \mathrm{~atm})$ & DMSO & 60 & 61 \\
\hline 17 & $\mathrm{Pd}(\mathrm{OAc})_{2}$ & $\mathrm{O}_{2}(1 \mathrm{~atm})$ & DMSO & 25 & 16 \\
\hline 18 & none & $\mathrm{O}_{2}(1 \mathrm{~atm})$ & DMSO & 120 & 0 \\
\hline 19 & none & none & DMSO & 120 & 0 \\
\hline 20 & $\mathrm{CuI}(0.2)$ & none & DMSO & 120 & 0 \\
\hline 21 & $\mathrm{FeCl}_{3}(0.3)$ & none & DMSO & 120 & 0 \\
\hline
\end{tabular}

${ }^{a}$ Reaction conditions: 1.0 eq. of 1a, 0.1 eq. of catalyst, 2 eq. of oxidant in $2 \mathrm{~mL}$ solvent were stirred for $16 \mathrm{~h} .{ }^{b}$ Isolated yield.

A variety of 3-arylsydnone (1) substrates were also investigated under the optimized conditions. Table 2 shows that bis-sydnones (2) were efficiently generated with moderate to 
good yields. The reaction proceeded efficiently when the aryl group of 3-arylsydnone (1) is a phenyl that carries an electron-donating group such as methoxy (1a) or methyl (1b and 1c), less so with an electron-withdrawing substituent such as halogen $(\mathbf{1} \mathbf{e}-\mathbf{1 h})$. The presence of an electron-donating group in the phenyl group of 3-arylsydnone (1a-c) increased the reaction yield compared with 1d (Table 2, entries 1-3). In contrast, the presence of one or two electronwithdrawing substituents in the phenyl group $(\mathbf{1} \mathbf{-}-\mathbf{1 h})$ generated relatively low yields of product 2 (Table 2, entries 5-8). Furthermore, higher yields were obtained when the substrates contained an electron-donating substituent at the para position than that at the meta position. This finding could be attributed to the ability of the electron-donating group at the para position to exert a stronger electron-donating inductive effect than the group at the meta position (Table 2, entries $2,3)$. This behavior was contrary to that of substrates (1) containing electron-withdrawing groups (Table 2, entries 6,7). We have also tested the reactivity of 3-(4-nitrophenyl)sydnone under the optimal conditions, but the desired product 3,3'-bis(4-nitrophenyl)-4,4'-bis-sydnone (2i) was not found (Table 2, entry 9). It appears that the strong electron-withdrawing inductive effect of the nitro group greatly reduced its reactivity.

Table 2. 3-Arylsydnones 1: scope in dimerization reaction (Scheme 1) ${ }^{a}$

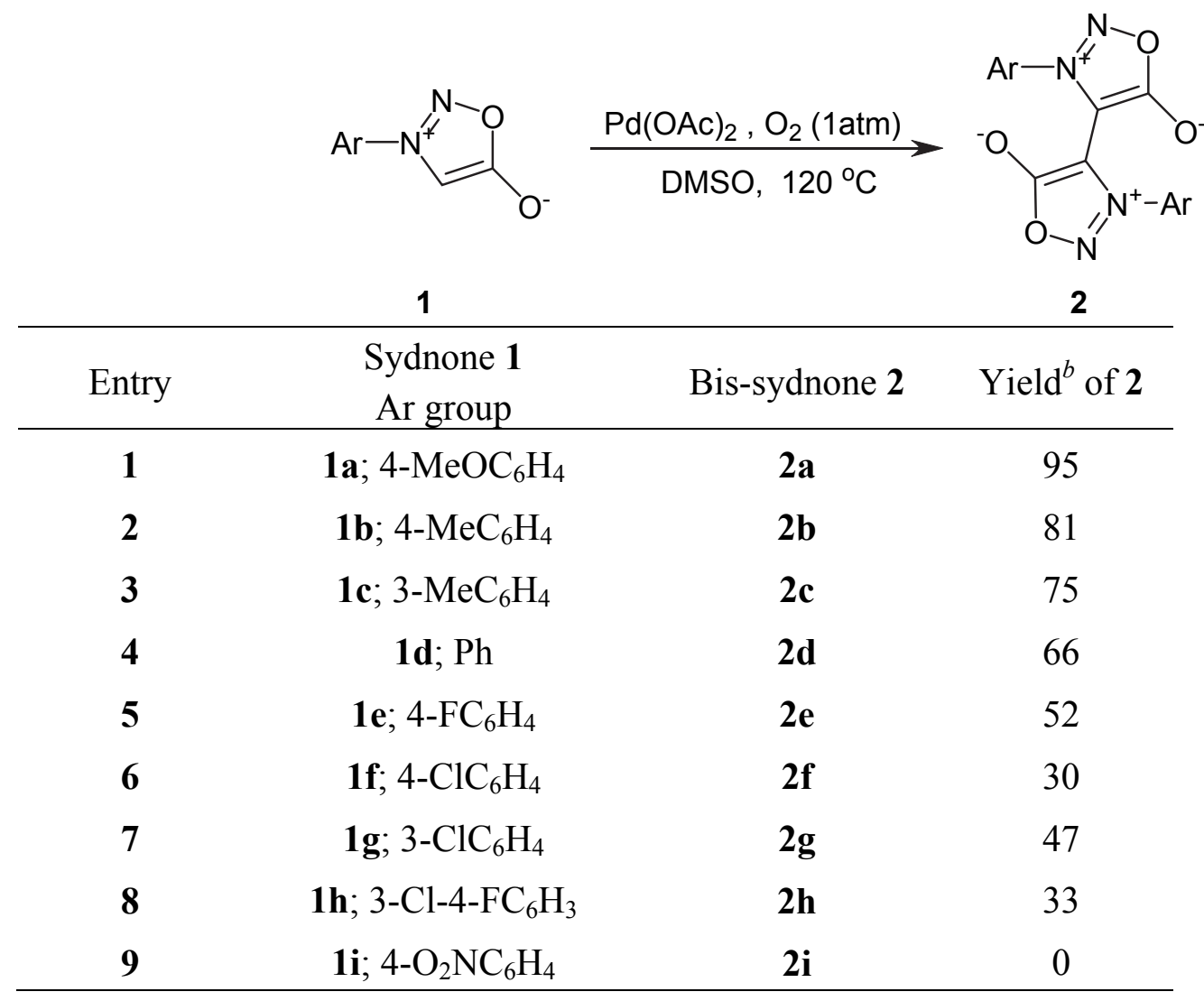

${ }^{a}$ Reaction conditions: 1.0 eq. of 1 and 0.1 eq. of $\mathrm{Pd}(\mathrm{OAc})_{2}$ in $2 \mathrm{~mL} \mathrm{DMSO}$ were stirred at $120^{\circ} \mathrm{C}$ for $16 \mathrm{~h}$ under $\mathrm{O}_{2}(1 \mathrm{~atm})$ atmosphere. ${ }^{b}$ Isolated yield. 
A plausible reaction mechanism for the synthesis of bis-sydnones (2) is shown in Scheme 2. The initial electronic attack of Pd (II) on 3-arylsydnone (1) and the subsequent deprotonation form species $\mathbf{A} .^{24-25}$ The resulting species $\mathbf{A}$ reacted with another 3-arylsydnone molecule to generate the corresponding intermediate $\mathbf{B}{ }^{26}$ The intermediate was then subjected to reductive elimination to synthesize the product $\mathbf{2}$ and generate $\mathrm{Pd}^{0}$. Finally, $\mathrm{Pd}(\mathrm{II})$ was regenerated through the oxidation of $\mathrm{Pd}^{0}$.

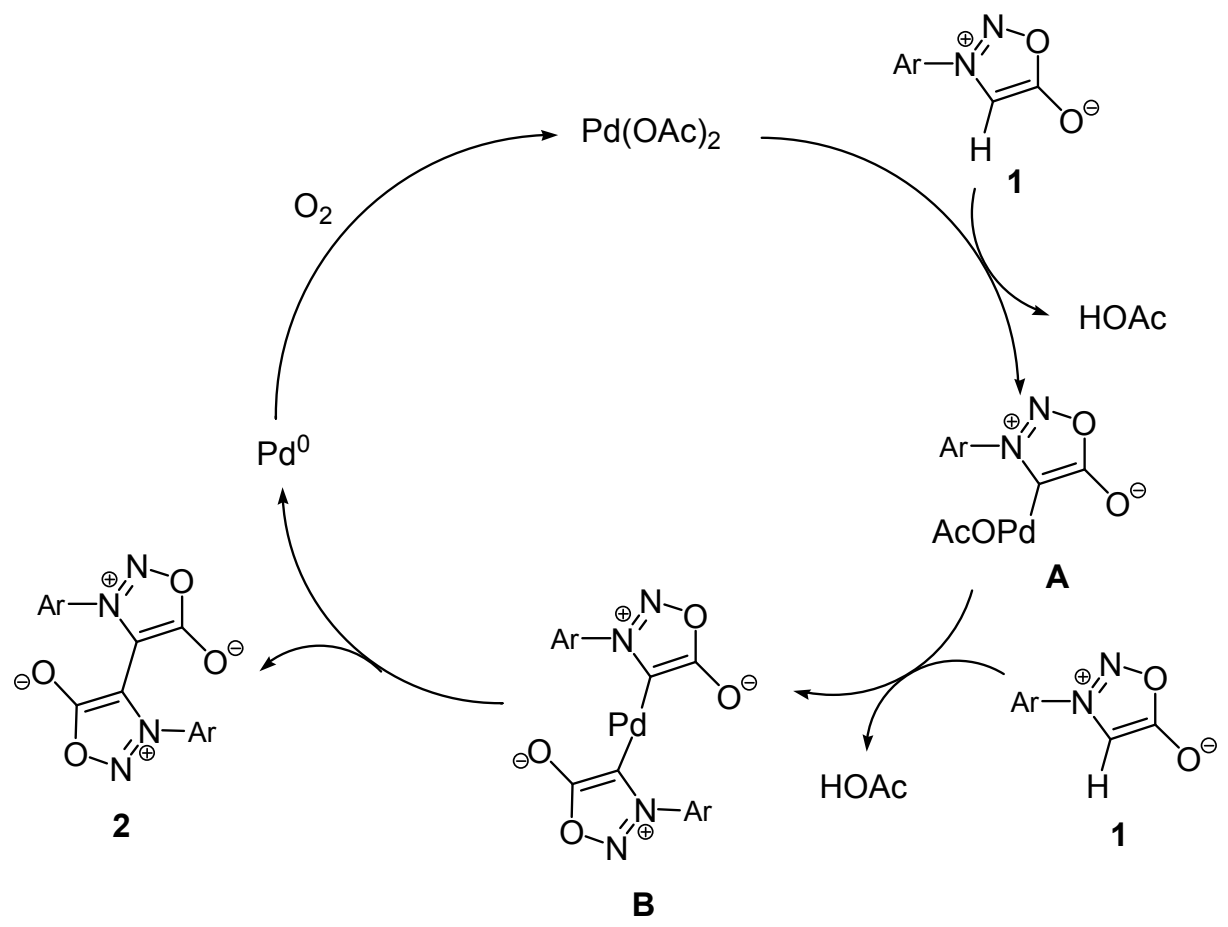

Scheme 2. Possible mechanism for the formation of 2.

\section{Conclusions}

In summary, we synthesized bis-sydnones in moderate to high yields through direct crossdehydrogenative coupling of 3-arylsydnones catalyzed by Pd under oxygen. It was convenient, efficient, and environment friendly and considered an atom- and step-economical process.

\section{Experimental Section}

General. All commercially available reagents and solvent were obtained from the commercial providers and used without further purification. Melting points were recorded using a WRS-2A melting point apparatus. IR spectra were obtained on a Nexus FT-IR spectrophotometer. ${ }^{1} \mathrm{H}$ NMR and ${ }^{13} \mathrm{C}$ NMR spectra were recorded using a Varian $400 \mathrm{MHz}$ spectrometer. Chemical 
shifts were reported relative to internal tetramethylsilane $(0.00 \mathrm{ppm})$ for ${ }^{1} \mathrm{H}$ and $\mathrm{CDCl}_{3}(77.0$ ppm) for ${ }^{13} \mathrm{C}$. High-resolution mass spectra were obtained on a Finnigan-NAT GC/MS/DS 8430 spectrometer. 3-Arylsydnones 1 were prepared according to literature procedures. ${ }^{27-29}$

General procedure for the synthesis of 2. A mixture of 3 -arylsydnone $(0.3 \mathrm{mmol})$ and $\mathrm{Pd}(\mathrm{OAc})_{2}(0.03 \mathrm{mmol})$ in dimethyl sulfoxide (DMSO, $\left.2 \mathrm{~mL}\right)$ was placed in a tube. The tube was equipped with a balloon filled with $\mathrm{O}_{2}(1 \mathrm{~atm})$. The mixture was stirred in an oil bath at $120{ }^{\circ} \mathrm{C}$ for $16 \mathrm{~h}$. After the reaction was completed (as monitored by TLC), the mixture was cooled to room temperature. The reaction mixture was then added with $30 \mathrm{~mL}$ of water and extracted with EtOAc three times $(20 \mathrm{~mL}$ of each extraction). The combined organic layers were washed with saturated brine, dried with anhydrous $\mathrm{Na}_{2} \mathrm{SO}_{4}$, and concentrated under reduced pressure. The resulting residue was purified through flash column chromatography (petroleum ether/ethyl acetate $=2: 1, \mathrm{v} / \mathrm{v})$ to yield bis-sydnones $(2)$.

3,3'-Bis(4-methoxyphenyl)-4,4'-bis-sydnone (2a). Yellow solid; yield 95\%; mp 194.4-195.1 ${ }^{\circ} \mathrm{C}$. ${ }^{1} \mathrm{H}$ NMR $\left(400 \mathrm{MHz}, \mathrm{CDCl}_{3}\right): \delta=3.86(\mathrm{~s}, 3 \mathrm{H}), 3.86$ (s, 3H), $7.00(\mathrm{dd}, J$ 9.2, $2.0 \mathrm{~Hz}, 4 \mathrm{H}), 7.41(\mathrm{dd}$, $J$ 9.2, $2.4 \mathrm{~Hz}, 4 \mathrm{H}) .{ }^{13} \mathrm{C}$ NMR (100MHz, $\left.\mathrm{CDCl}_{3}\right): \delta=55.8(2 \mathrm{C}), 93.2$ (2C), $115.3(4 \mathrm{C}), 125.2(4 \mathrm{C})$, 126.1 (2C), 126.7 (2C), 166.6 (2C). IR (KBr): 3077, 2926, 2857, 1757, 1598, 1506, 1447, 1405, 1254, $1175,832 \mathrm{~cm}^{-1}$.HR-MS(ESI): $m / z$ calcd for $\mathrm{C}_{18} \mathrm{H}_{14} \mathrm{~N}_{4} \mathrm{NaO}_{6}: 405.0805[\mathrm{M}+\mathrm{Na}]^{+}$; found: 405.0801 .

3,3'-Di-p-tolyl-4,4'-bis-sydnone (2b). Tan solid; yield 81\%; mp 189.9-190.6 ${ }^{\circ} \mathrm{C} .{ }^{1} \mathrm{H}$ NMR (400 $\left.\mathrm{MHz}, \mathrm{CDCl}_{3}\right): \delta=2.44(\mathrm{~s}, 3 \mathrm{H}), 2.44(\mathrm{~s}, 3 \mathrm{H}), 7.33(\mathrm{~d}, J 9.2 \mathrm{~Hz}, 4 \mathrm{H}), 7.41(\mathrm{~d}, J 8.4 \mathrm{~Hz}, 4 \mathrm{H}) .{ }^{13} \mathrm{C}$ NMR (100MHz, $\left.\mathrm{CDCl}_{3}\right): \delta=21.5$ (2C), 93.2 (2C), 123.5 (4C), 130.8 (4C), 131.2 (2C), 143.8 (2C), 166.4 (2C). IR (KBr): 3070, 2925, 2860, 1751, 1606, 1503, 1434, 1282, 1183, $818 \mathrm{~cm}^{-1}$. HR-MS(ESI): $m / z$ calcd for $\mathrm{C}_{18} \mathrm{H}_{14} \mathrm{~N}_{4} \mathrm{NaO}_{4}: 373.0907$ [M+Na] $]^{+}$; found: 373.0902 .

3,3'-Di-m-tolyl-4,4'-bis-sydnone (2c). Tan solid; yield 75\%; mp 172.0-172.9 ${ }^{\circ} \mathrm{C}$. ${ }^{1} \mathrm{H}$ NMR (400 $\left.\mathrm{MHz}_{\mathrm{CDCl}}\right): \delta=2.41(\mathrm{~s}, 6 \mathrm{H}), 7.22(\mathrm{~d}, J 7.2 \mathrm{~Hz}, 2 \mathrm{H}), 7.25(\mathrm{~s}, 2 \mathrm{H}), 7.38-7.48(\mathrm{~m}, 4 \mathrm{H}) .{ }^{13} \mathrm{C} \mathrm{NMR}$ $\left(100 \mathrm{MHz}, \mathrm{CDCl}_{3}\right): \delta=21.3(2 \mathrm{C}), 93.1(2 \mathrm{C}), 120.7$ (2C), 124.3 (2C), $130.0(2 \mathrm{C}), 133.6(2 \mathrm{C})$, 133.6 (2C),141.0 (2C), 166.3 (2C). IR (KBr): 3069, 2925, 2860, 1749, 1622, 1466, 1388, 1292 , 1183, $791 \mathrm{~cm}^{-1}$. HR-MS(ESI): $m / z$ calcd for $\mathrm{C}_{18} \mathrm{H}_{14} \mathrm{~N}_{4} \mathrm{NaO}_{4}: 373.0907[\mathrm{M}+\mathrm{Na}]^{+}$; found: 373.0903

3,3'-Diphenyl-4,4'-bis-sydnone (2d). Tan solid; yield 66\%; mp 181.4-182.5 ${ }^{\circ} \mathrm{C}$. ${ }^{1} \mathrm{H}$ NMR (400 $\left.\mathrm{MHz}, \mathrm{CDCl}_{3}\right): \delta=7.47(\mathrm{~d}, J 8.0 \mathrm{~Hz}, 4 \mathrm{H}), 7.57(\mathrm{t}, J 7.8 \mathrm{~Hz}, 4 \mathrm{H}), 7.67(\mathrm{~d}, J 7.4 \mathrm{~Hz}, 2 \mathrm{H}) .{ }^{13} \mathrm{C} \mathrm{NMR}$ $\left(100 \mathrm{MHz}, \mathrm{CDCl}_{3}\right): \delta=93.2(2 \mathrm{C}), 123.8(4 \mathrm{C}), 130.3$ (4C), $132.9(2 \mathrm{C}), 133.6(2 \mathrm{C}), 166.3$ (2C). IR (KBr): 3099, 1750, 1645, 1481, 1412, 1276, 1089, $721 \mathrm{~cm}^{-1}$. HR-MS(ESI): $\mathrm{m} / z$ calcd for $\mathrm{C}_{16} \mathrm{H}_{10} \mathrm{~N}_{4} \mathrm{NaO}_{4}: 345.0594[\mathrm{M}+\mathrm{Na}]^{+}$; found: 345.0590 .

3,3'-Bis-(4-fluorophenyl)-4,4'-bis-sydnone (2e). Tan solid; yield 52\%; mp 207.7-208.9 ${ }^{\circ} \mathrm{C}$. ${ }^{1} \mathrm{H}$ NMR (400 MHz, $\left.\mathrm{CDCl}_{3}\right): \delta=7.25-7.32(\mathrm{~m}, 4 \mathrm{H}), 7.52-7.60(\mathrm{~m}, 4 \mathrm{H}) .{ }^{13} \mathrm{C} \mathrm{NMR}(100 \mathrm{MHz}$, $\left.\mathrm{CDCl}_{3}\right): \delta=93.3(2 \mathrm{C}), 117.7$ (d, $\left.J 23.6 \mathrm{~Hz}, 4 \mathrm{C}\right), 126.3$ (d, $\left.J 9.4 \mathrm{~Hz}, 4 \mathrm{C}\right), 129.6$ (2C), 164.8 (d, $J$ $249.2 \mathrm{~Hz}, 2 \mathrm{C}), 166.1$ (2C). IR (KBr): 3086, 1768, 1598, 1504, 1408, 1234, 1096, $842 \mathrm{~cm}^{-1}$. HRMS(ESI): $m / z$ calcd for $\mathrm{C}_{16} \mathrm{H}_{8} \mathrm{~F}_{2} \mathrm{~N}_{4} \mathrm{NaO}_{4}: 381.0406[\mathrm{M}+\mathrm{Na}]^{+}$; found: 381.0403 . 
3,3'-Bis-(4-chlorophenyl)-4,4'-bis-sydnone (2f). Tan solid; yield $30 \%$; mp $213.8-214.4^{\circ} \mathrm{C} .{ }^{1} \mathrm{H}$ NMR $\left(400 \mathrm{MHz}, \mathrm{CDCl}_{3}\right): \delta=7.50(\mathrm{~d}, J 8.8 \mathrm{~Hz}, 4 \mathrm{H}), 7.56(\mathrm{~d}, J 8.4 \mathrm{~Hz}, 4 \mathrm{H}) .{ }^{13} \mathrm{C} \mathrm{NMR}(100 \mathrm{MHz}$, $\mathrm{CDCl}_{3}$ ): $\delta=93.1$ (2C), 125.2 (4C), 130.7 (4C), 131.9 (2C), 139.7 (2C), 166.0 (2C). IR (KBr): $3062,1757,1646,1465,1278,1085,757 \mathrm{~cm}^{-1}$. HR-MS(ESI): $m / z$ calcd for $\mathrm{C}_{16} \mathrm{H}_{8}{ }^{35} \mathrm{Cl}_{2} \mathrm{~N}_{4} \mathrm{NaO}_{4}$ : $412.9815[\mathrm{M}+\mathrm{Na}]^{+}$; found: 412.9810

3,3'-Bis-(3-chlorophenyl)-4,4'-bis-sydnone (2g). Light yellow solid; yield 47\%; mp 105.6106.9 ${ }^{\circ} \mathrm{C} .{ }^{1} \mathrm{H}$ NMR $\left(400 \mathrm{MHz}, \mathrm{CDCl}_{3}\right): \delta=7.46-7.52(\mathrm{~m}, 4 \mathrm{H}), 7.55(\mathrm{t}, J 8.2 \mathrm{~Hz}, 2 \mathrm{H}), 7.67(\mathrm{~d}, J$ $8.0 \mathrm{~Hz}, 2 \mathrm{H}) .{ }^{13} \mathrm{C}$ NMR $\left(100 \mathrm{MHz}, \mathrm{CDCl}_{3}\right): \delta=90.7(2 \mathrm{C}), 105.0(2 \mathrm{C}), 122.1(2 \mathrm{C}), 124.2(2 \mathrm{C})$, 131.5 (2C), 133.3 (2C), 136.4 (2C), 162.0 (2C). IR (KBr): 3080, 1799, 1589, 1461, 1295, 1085, $785725 \mathrm{~cm}^{-1}$. HR-MS(ESI): $\mathrm{m} / z$ calcd for $\mathrm{C}_{16} \mathrm{H}_{8}{ }^{35} \mathrm{Cl}_{2} \mathrm{~N}_{4} \mathrm{NaO}_{4}: 412.9815[\mathrm{M}+\mathrm{Na}]^{+}$; found: 412.9811

3,3'-Bis-(3-chloro-4-fluorophenyl)-4,4'-bis-sydnone (2h). Yellow solid; yield 33\%; mp 119.8$120.7^{\circ} \mathrm{C} .{ }^{1} \mathrm{H}$ NMR $\left(400 \mathrm{MHz}, \mathrm{CDCl}_{3}\right): \delta=7.39(\mathrm{t}, J 8.2 \mathrm{~Hz}, 2 \mathrm{H}), 7.53-7.61(\mathrm{~m}, 2 \mathrm{H}), 7.65-7.72$ $(\mathrm{m}, 2 \mathrm{H}) .{ }^{13} \mathrm{C} \mathrm{NMR}\left(100 \mathrm{MHz}, \mathrm{CDCl}_{3}\right): \delta=93.3(2 \mathrm{C}), 118.6(\mathrm{~d}, J 23.2 \mathrm{~Hz}, 2 \mathrm{C}), 124.0(2 \mathrm{C}), 124.2$ (d, J 8.4 Hz, 2C), 126.8 (2C), 129.7 (d, J 3.1 Hz, 2C), 160.7 (d, J 257.4 Hz, 2C), 165.7 (2C). IR (KBr): 3090, 1800, 1639, 1496, 1265, 1074, $712 \mathrm{~cm}^{-1}$. HR-MS(ESI): $\mathrm{m} / z$ calcd for $\mathrm{C}_{16} \mathrm{H}_{6}{ }^{35} \mathrm{Cl}_{2}{ }^{19} \mathrm{~F}_{2} \mathrm{~N}_{4} \mathrm{NaO}_{4}: 448.9626[\mathrm{M}+\mathrm{Na}]^{+}$; found: 448.9622

\section{Acknowledgements}

This work was supported by the Natural Science Foundation of China (No. 21401078). We would like to thank the Testing and Analysis Center of Jiaxing University and the Center for Instrumental Analysis, Tongji University, China.

\section{References}

1. Browne, D. L.; Harrity J. P. A. Tetrahedron 2010, 66, 553. http://dx.doi.org/10.1016/j.tet.2009.10.085

2. Kavali, J. R.; Badami, B. V. Farmaco 2000, 55, 406. http://dx.doi.org/10.1016/S0014-827X(00)00061-6

3. Hill, J. B.; Ray, R. E.; Wagner, H.; Aspinall, R. L. J. Med. Chem. 1975, 18, 50. http://dx.doi.org/10.1021/jm00235a011

4. Kier, L. B.; Roche, E. B. J. Pharm. Sci. 1967, 56, 149. http://dx.doi.org/10.1002/jps.2600560202

5. McCaustland, D. J.; Burton, W. H.; Cheng, C. C. J. Heterocycl. Chem. 1971, 8, 89. http://dx.doi.org/10.1002/jhet.5570080117

6. Satyanarayana, K.; Rao, M. N. A. J. Pharm. Sci. 1995, 84, 263. http://dx.doi.org/10.1002/jps.2600840228 
7. Dunkley, C. S.; Thomas, C. J. Bioorg. Med. Chem. Lett. 2003, 13, 2899. http://dx.doi.org/10.1016/S0960-894X(03)00487-6

8. Moustafa, M. A.; Gineinah, M. M.; Nasr, M. N.; Bayoumi, W. A. H. Arch. Pharm. (Weinheim) 2004, 337, 164. http://dx.doi.org/10.1002/ardp.200300814

9. Geoffroy, I.; Carre, B.; Lemordant, D. ITE Lett. Batter., New Technol. Med. 2000, 1, 20.

10. Chan, W. L.; Zhang, W. H.; Szeto, Y. S. Mater. Lett. 2000, 42, 280. http://dx.doi.org/10.1016/S0167-577X(99)00196-2

11. Sasaki, Y.; Hirobomi, O.; Handa, M. Nippon Kagaku Kaishi 1993, 1217. http://dx.doi.org/10.1246/nikkashi.1993.1217

12. Tien, H. J.; Yeh, M. Y. J. Chin. Chem. Soc. 1977, 24, 123. http://dx.doi.org/10.1002/jccs.197700020

13. Tien, H. J.; Lee, Y. K. J. Chin. Chem. Soc 1988, 35, 63. http://dx.doi.org/10.1002/jccs.198800010

14. Kalinin, V. N.; Min, S. F. J. Organomet. Chem. 1988, 352, C34. http://dx.doi.org/10.1016/0022-328X(88)83049-3

15. Wu, C.; Li, P.; Fang, Y.; Zhao, J.; Xue, W.; Li, Y.;Larock, R. C.; Shi, F. Tetrahedron Lett. 2011, 52, 3797. http://dx.doi.org/10.1016/j.tetlet.2011.05.058

16. Brown, A. W.; Harrity, J. P. A. J. Org. Chem. 2015, 80, 2467. http://dx.doi.org/10.1021/acs.joc.5b00143

17. Yang, Y.; Kuang, C. Eur. J. Org. Chem. 2014, 7810. http://dx.doi.org/10.1002/ejoc.201403211

18. Chen, X.; Engle, K. M.; Wang, D.; Yu, J. Angew. Chem. Int. Ed. 2009, 48, 5094. http://dx.doi.org/10.1002/anie.200806273

19. Sun, C.; Li, B.; Shi, Z. Chem. Commun. 2010, 46, 677. http://dx.doi.org/10.1039/b908581e

20. Fan, M.; Ma, D. Angew. Chem. Int. Ed. 2013, 52, 12152. http://dx.doi.org/10.1002/anie.201306583

21. Baumann, C. G.; Ornellas, S. D.; Reeds, J. P.; Storr, T. E.; Williams, T. J.; Fairlamb, I. J. S. Tetrahedron 2014, 70, 6174. http://dx.doi.org/10.1016/j.tet.2014.06.002

22. Li, C. Acc. Chem. Res. 2008, 42, 335. http://dx.doi.org/10.1021/ar800164n

23. Wang, Z.; Li, K.; Zhao, D.; Lan, J.; You, J. Angew. Chem. Int. Ed. 2011, 50, 1.

24. Ge, H.; Niphakis, M. J.; Georg, G. I. J. Am. Chem. Soc. 2008, 130, 3708. http://dx.doi.org/10.1021/ja710221c

25. Lu, Z.; Luo, F.; Wang, L.; Zhu, G. J. Org. Chem. 2013, 78, 10894. http://dx.doi.org/10.1021/jo4018793 
26. Liang, P.; Xiong, H.; Guo, H.; Yin, G. Catal. Commun. 2010, 11, 560. http://dx.doi.org/10.1016/j.catcom.2009.12.019

27. Rai, N. S.; Kalluraya, B.; Lingappa, B.; Shenoy, S.; Puranic, V. G. Eur. J. Med. Chem. 2008, $43,1715$.

http://dx.doi.org/10.1016/j.ejmech.2007.08.002

28. Asundaria, S. T.; Patel, N. S.; Patel, K. C. Org. Commun. 2010, 3, 30.

29. Fang, Y.; Wu, C.; Larock, R. C.; Shi, F. J. Org. Chem. 2011, 76, 8840. http://dx.doi.org/10.1021/jo201605v 